\section{Los (des)caminos de la formación profesional del psicólogo en Brasil para la actuación en la salud publica ${ }^{1}$}

Magda Dimenstein ${ }^{2}$

Palabras clave: educación de profesionales de la salud, psicología, atención primaria, Brasil.

1 Este trabajo fue presentado en la 2. ${ }^{a}$ Convención Internacional de Educación Superior, La Habana, Cuba, 2000.

2 Universidade Federal de Rio Grande do Norte, Natal/RN, Brasil. Dirección postal: Universidade Federal do Rio Grande do Norte, Depto. de Psicologia, Centro de Ciências Humanas, Letras e Artes, Campus Universitário, Natal, RN, CEP: 59.078-970, Brasil. Teléfono y fax: 55-084.215.3589. Correo electrónico: magdad@uol.com.br
La formación académica que reciben los psicólogos en el Brasil no los prepara adecuadamente para responder a las demandas de la atención primaria de la salud (APS), reconocer las necesidades y recursos de la población usuaria de los servicios públicos, ni buscar maneras de remediar las desigualdades sociales. El objetivo de este trabajo es destacar las dificultades que aquejan a la atención psicológica en las unidades básicas de salud (UBS) de Brasil como resultado de esa formación inadecuada y orientada hacia modelos de actuación limitados al sector de la salud y poco adaptados a la demanda de la clientela.

La precaria formación del psicólogo para actuar en las UBS determina tanto la falta de definición de sus funciones en la red básica de salud como el predominio de actividades típicas del modelo clásico de la psicología clínica liberal y privada en los puestos, centros y ambulatorios, la cual no toma en cuenta los objetivos de los mismos ni las demandas de la población. De esta manera, se puede decir que la formación del psicólogo es uno de los principales elementos que definen sus modelos de actuación en las UBS, y en consecuencia, la descontextualización de sus prácticas y la pobreza de los resultados alcanzados. Así, el propósito de este trabajo es abrir un camino para la discusión de dos cuestiones fundamentales:

- ¿Cuál es la formación académica necesaria para lograr una intervención más definida y adecuada del psicólogo en el ámbito de las UBS?

- ¿Cuál ha sido la respuesta de las universidades frente a esa exigencia en el sector de la salud pública? $(1,2)$

Cabe resaltar que este texto no es un relato de investigación. Se trata de un conjunto de reflexiones ancladas en la experiencia profesional y docente, así como en los resultados y el análisis de dos investigaciones realizadas por la autora con psicólogos que trabajan en la red básica de salud de dos ciudades del nordeste brasileño: Nata (Rio Grande do Norte) y Teresina (Piauí). Los datos referentes a los psicólogos de Natal son fruto de una investigación aún en curso en la que toman parte profesionales de la salud de distintas especialidades y que versa sobre el proceso de trabajo y la formación académica. En cuanto a los profesionales de Teresina, el mapeo realizado es una etapa del trabajo de campo que la autora llevó 
a cabo en las UBS sobre la evaluación de los servicios públicos de psicología. Los resultados de estas investigaciones y los procedimientos de definición de la muestra aparecen en dos publicaciones $(3,4)$.

Discutir esta cuestión es importante dado que, aunque la especialidad es numéricamente escasa comparada con las otras profesiones de la salud, la presencia del psicólogo ya se hace sentir en los equipos de salud en todo el Brasil, y su contratación en el sector muestra un crecimiento superior a la media cuando se compara con otras especialidades profesionales. Es decir, al psicólogo ya se le considera parte integrante de los equipos de salud. A pesar de ello, no parece haber preocupación en las instituciones de enseñanza ni entre los profesionales por reflexionar acerca de lo que se ha venido realizando en ese campo en cuanto a la capacitación práctica del psicólogo para el desempeño de estas actividades, que se relacionan directamente con el cuidado o las intervenciones de orden sanitario. Asimismo, son conocidas las grandes dificultades que enfrenta el psicólogo para integrarse a los equipos y programas de salud. Como ejemplo tenemos el caso de la ausencia de psicólogos en el equipo del Programa de Salud de la Familia (PSF), implantado por el Ministerio de Salud de Brasil.

Es común que los profesionales hablen de su trabajo cotidiano en la red pública de salud con un cierto "malestar" que se traduce en un estado de desorientación o paralización ante los problemas planteados, malestar que se refleja en la práctica y compromete la eficacia de sus intervenciones. Por otro lado, se sabe que los servicios de psicología registran considerables tasas de abandono prematuro de los tratamientos por parte de los usuarios, o sea que hay un alto índice de falta de adhesión a las propuestas terapéuticas de los psicólogos. Factores como la degradación del salario, la inoperancia de los equipos de salud, la precariedad de la estructura física y la falta de aporte de material y apoyo por parte de la dirección de las instituciones de salud también generan malestar entre los psicólogos, pues se identifican como factores que desvalorizan al profesional. Estos factores asumen proporciones tan importantes que terminan por conformar prácticas movidas por la ley del "menor esfuerzo posible", sin compromiso con un proyecto más amplio de rescate de la ciudadanía, es decir, con prácticas más solidarias y éticamente humanas (5).

Es necesario aceptar que todas estas dificultades que el psicólogo enfrenta en el sector público inciden en su funcionamiento dentro de las instituciones de salud. Actualmente se considera que el retraso salarial es notorio, que la falta de estructura física y material y principalmente de apoyo institucional inciden sobre la calidad de la asistencia prestada por los profesionales, y que la dificultad que tienen los psicólogos para integrarse a los equipos y programas de salud se debe en parte a la propia segmentación de los saberes, que es histórica, y a los aspectos corporativistas de las diversas especialidades profesionales. La intención no es responsabilizar al psicólogo por el fracaso de los equipos multidisciplinarios que tan caros le salen al sistema de salud de Brasil. No obstante, se entiende que no podíamos limitar nuestra investigación a la práctica de los psicólogos en el ámbito de la salud y a las dificultades que ahí encuentran sin referirnos a ciertas características propias de la formación que el psicólogo trae consigo y que producen cierta subjetividad, es decir, una determinada manera de ver y de pensar, de organización y de representación. Todas estas producen una identidad y una cultura profesional muy específicas que condicionan su actuación, los espacios concretos donde puede actuar, la elección de la clientela y las necesidades que puede atender, las técnicas y procedimientos que utilizará, así como la representación social de la profesión para los legos y para el propio psicólogo.

No se puede dejar de considerar, por tanto, que la dificultad del psicólogo para insertarse en los equipos de salud también está asociada con la rigidez de los conocimientos especializados que trae de la universidad y con la resistencia a adaptar su postura técnica. En otras palabras, los problemas a los que se enfrentan los psicólogos en el ámbito de la asistencia pública de la salud no pueden atribuirse por entero al tipo de demanda (que se diferencia de la de la clínica privada) y a las dificultades que plantea la clientela de bajos recursos que acude al sector público, ni tampoco a los problemas relacionados con la estructura y la organización de los servicios. Se entiende que la raíz de estas dificultades debe buscarse en los aportes teóricos y prácticos en los que se basan los modelos de actuación de los psicólogos, en su identidad y cultura profesional y en las expectativas que la sociedad tiene con relación a su papel en el ámbito de la salud. Es ahí donde se puede encontrar la raíz de los fracasos vividos por los psicólogos y de su dificultad para pensar y ofrecer alternativas de trabajo acordes con las necesidades y características de la población, es decir, formas de actuar más amplias que las que suelen aprenderse tradicionalmente durante su formación académica (5).

De hecho, la mayor parte de los trabajos producidos en el país en torno a esta cuestión apuntan a una transposición pura y simple del modelo clínico de actuación privada (la 
psicoterapia individual de base psicoanalítica) hacia el sector público, que prescinde de los objetivos de este último y de las características de la población atendida. En función de esto, no es raro que en el sector público se dé prioridad al uso de la técnica grupal como posible solución a las dificultades que los psicólogos enfrentan, en el supuesto de que sería más adecuada y beneficiaría a un mayor número de personas que la atención individual. Entretanto, se percibe que con la técnica grupal, supuestamente más adecuada, los grupos de pacientes no se mantienen. Lo que se plantea no es, por consiguiente, si la técnica grupal es mejor que la individual para la clientela que frecuenta los servicios públicos de salud, sino si tanto la una como la otra es apropiada en este contexto y si la función del psicólogo es necesariamente practicar la psicoterapia. Se considera equivocado enfocar el frecuente abandono de los tratamientos como algo reducible a una técnica determinada sin antes haber cuestionado los supuestos básicos sobre los cuales se apoyan esas técnicas y los marcos teóricos que las sustentan. No se puede dar por sentado que los procedimientos psicoterapéuticos, sea cual fuere su naturaleza, son eficaces en sí mismos; no cuestionar la eficacia de una técnica equivale a creer que todo es legítimo en este campo y que es posible utilizarla en los más diferentes contextos, sin tener en cuenta los posibles efectos yatrógenos de esos procedimientos.

Ciertamente, la formación del psicólogo tiene mucho que ver con esto. El campo de la psicología está imbuido predominantemente por teorías esencialistas y universalistas en torno a la "naturaleza" humana, esto es, por teorías según las cuales el ser humano es invariable y universal. Por otro lado, se utilizan acríticamente modelos teóricos y prácticos importados de otras realidades sin tener en cuenta la inexistencia de sujetos abstractos y ahistóricos. Nuestra enseñanza valoriza demasiado al psicólogo como profesional liberal, de manera que esta representación constituye un fuerte polo de atracción para quienes eligen la profesión. Como dicha enseñanza está fuertemente teñida por el referencial psicoanalítico, nuestros currículos reflejan y producen un modelo de actuación profesional (la psicoterapia individual de base psicoanalítica) que se tornó hegemónico en nuestro campo, proporcionando definiciones extremamente limitadas de lo que es una intervención psicológica y de cuál es el campo concreto de la práctica del psicólogo (6). Es importante también apuntar que en el campo psicológico se trabaja con una concepción muy particular de subjetividad, el "sujeto psicológico", desarrollado a partir del ideal individualista predominante en la sociedad occidental moderna y engendrado por los propios saberes "psi", lo cual también puede considerarse como uno de los efectos de su difusión. La hegemonía de este modelo de subjetividad, que está presente en los saberes y prácticas "psi", es problemática en la medida en que no se desarrolla en el contexto del usuario, es decir, en la medida en que se considera el único modelo de subjetividad posible, menospreciando así la complejidad del proceso de construir la subjetividad y los múltiples factores que inciden en ella. Entretanto, la sociología y la antropología vienen mostrando desde hace ya cierto tiempo que no existe una "naturaleza" humana universal $y$, consecuentemente, que tampoco son universales ciertos modelos y representaciones presen-tes propios de los saberes "psi", tales como los de salud-enfermedad, lo normal-patológico, el cuerpo, la muerte, la sexualidad, etc., y que esos modelos pueden variar no solo de una cultura a otra y a través de los tiempos, sino también entre las diferentes clases sociales $(7,8)$. Cuando los psicólogos trasladan sus técnicas y teorías psicológicas a la atención de los usuarios que frecuentan las instituciones públicas de salud, quienes en general pertenecen a las clases populares, parten del supuesto de que esta población comparte la misma visión del mundo, las mismas representaciones antes mencionadas y el mismo modelo de subjetividad que brindan sustento a sus formas de actuación. Sin embargo, muchos autores ya han observado que existen diferencias culturales entre terapeutas y pacientes, que estos no tienen las mismas representaciones sociales ni el mismo modelo de subjetividad. Así, se establece una verdadera ruptura entre las propuestas de los profesionales de la psicología y los objetivos de los usuarios, lo que deriva en un gran número de abandonos del tratamiento y en la reducida eficacia de las terapias utilizadas por los psicólogos.

En psicología, los currículos se presentan muchas veces como una serie de disciplinas estancadas y descontextualizadas que terminan por aumentar el aislamiento del psicólogo en el ámbito de la salud. Pensamos que la solución no debe buscarse en nuevas organizaciones profesionales, a pesar de que sería beneficioso realizar algunos cambios en el orden actual de las distintas disciplinas. Antes se debería repensar la actitud pasiva que tenemos, sabiéndonos consumidores acríticos de cosas creadas en otras realidades. No obstante, el mayor problema no es la importación de la teoría en sí, sino el pensar que tiene carácter universal, es decir, que se pueden aplicar de manera generalizada, rígida y estricta los procedimientos clínicos a todos y cada uno de los mismos grupos sociales y culturales, y que dichos procedimientos tienen una eficacia intrínseca. Se puede decir que en 
Brasil una parte de los cursos de psicología proporcionan al estudiante lo siguiente:

1. Patrones técnicos de cuidado y de reproducción de la despolitización del psicólogo que aumentan la distancia entre la psicología y los problemas objetivos de la sociedad, generando una ruptura entre las necesidades sociales y el saber técnico ofrecido al psicólogo, y un desconocimiento de la función ideológica de su trabajo (adaptación y ajuste);

2. una visión fragmentada, con un currículo inadecuado para la adquisición de conocimientos específicos para la actuación en el ámbito de la salud. Además de eso, docentes sin experiencia de trabajo en las UBS y en los problemas de la salud pública brasileña, sin interés en estudios, investigaciones y prácticas supervisadas en el área;

3. una imagen social de la profesión imbuida por el ideal profesional liberal (psicoterapeuta) con un modelo de actuación reducido y una visión elitista del mundo.

Tal formación se configura como un fuerte componente de la determinación de un perfil profesional inadecuado para la realidad de salud del país y el compromiso con los principios básicos que orientan el SUS, que es la forma de provisión de servicios propuesta actualmente para el sector público de Brasil. Nuestro sistema está anclado en algunos principios fundamentales que implican un nuevo modelo asistencial: la salud comprendida como el efecto de las condiciones concretas de vida y no solo como la ausencia de enfermedad o como un estado abstracto de normalidad; las intervenciones y los servicios deben ofrecerse de acuerdo con las necesidades de la población. Además de eso, se parte del principio de que el sujeto no es pasivo en el proceso "salud y enfermedad", de que no es un mero objeto de las intervenciones sino un actor social igualmente responsable dentro del ámbito de la salud pública (9).

Ese modelo de atención y cuidado implica un desafío para la psicología en lo atinente a este campo del saber, así como para los profesionales en lo que respecta a sus instrumentos teóricos y prácticos, es decir, implica un cambio en el perfil profesional hegemónico en que el psicólogo es identificado como psicoterapeuta. También implica una reorientación del modelo asistencial individual hacia modelos de explicación y análisis más complejos y formas de intervención más amplias y participativas. Eso apunta necesariamente a un concepto distinto de contrato y vínculo terapéutico, basado especialmente en el compromiso ético con los usuarios de los servicios públicos de salud. Los profesionales de la psicología necesitan ofrecer servicios de acuerdo a las necesidades y prioridades de salud de la población, tomando como base su realidad epidemiológica; deberán desarrollar acciones intersectoriales y de colaboración con otros profesionales que formen parte del equipo de salud, priorizar mecanismos de decisión horizontales y evitar las relaciones verticales y jerarquizadas que dificultan el intercambio de experiencias entre las diferentes disciplinas. Ciertamente esto requiere modelos democráticos de administración de los servicios de salud en los cuales la evaluación sea un proceso permanente que sirva de base a la planificación. Será necesario revisar el concepto de intervención psicológica, así como las teorías y técnicas privilegiadas, incluir conocimientos de otros campos del saber, discutir aspectos de la cultura y la identidad profesional, y buscar modelos más flexibles.

Para la materialización de ese ideario, esto es, para una intervención del psicólogo más definida y adecuada a las UBS, su formación debe posibilitar la construcción de un modelo ampliado de desempeño profesional, tanto en sus aspectos teóricos y prácticos como en relación con su función social, que lo capacite para prestar una mayor variedad de servicios, promover una postura crítica con respecto a los conocimientos generados en otros contextos, e imponer la reflexión y sistematización del conocimiento que se va generando a partir de las nuevas prácticas y de la atención de la nueva clientela. Además, la formación del psicólogo debe fomentar una revisión de los conceptos hegemónicos acerca de la subjetividad y el aparato psíquico en función de los diferentes procesos de socialización e inserción cultural de los individuos, y de la concepción medicalizada de la salud y la enfermedad. Así, se puede afirmar que el trabajo del psicólogo en el SUS debe estar centrado en algunos puntos fundamentales:

- Saber identificar cuáles son los problemas que requieren atención prioritaria dentro de la comunidad en la que se trabaja, es decir, conocer los problemas más frecuentes, determinar las necesidades de salud de la comunidad y ser un detector de problemas. Es importante estimular la participación de los usuarios como identificadores de problemas y multiplicadores de soluciones. No obstante, la necesidad de salud no puede ser entendida como un concepto unívoco, pues no se traduce de la misma forma en cada clase, fracción de clase o grupo social. Se entiende, además, que las diferentes concepciones de lo que constituye una necesidad de salud repercuten en el modo en que las 
personas se relacionan con el servicio de salud y en lo que definen como prioridades sanitarias.

- Desarrollar actividades de promoción de la salud en contextos particulares. Mediante la educación en materia de salud se busca comprender las causas de los problemas de salud y hallarles soluciones mediante un intercambio entre el saber científico y el popular, es decir, un diálogo, un traspaso de saberes. Tal postura difiere del concepto de educación caracterizado por la búsqueda de una modificación de hábitos y comportamientos perjudiciales por imposición de los conocimientos del técnico o experto en el área de la salud. En cambio, dicha postura promueve el desarrollo de actividades fuera del ámbito institucional, aspira a adecuar y flexibilizar el entorno terapéutico (setting) en sus intervenciones, y se preocupa por los aspectos éticos de los compromisos sociales y políticos (10).

- Evaluar continuamente los resultados de su intervención y la calidad de la atención prestada. Para eso es necesario desarrollar la metodología y los indicadores adecuados para los servicios de psicología, lo cual permite proponer por último modificaciones en la práctica y en los servicios.

Finalmente, creemos que es necesario cuestionar las formas instituidas en los currículos, las cuales producen una acumulación de conocimientos que se consideran absolutos y que se utilizan como instrumento de poder y control social. Cualquier transformación en este campo exige, pues, la deconstrucción de sus supuestos básicos, de una visión naturalista y privatista del hombre. Así, los psicólogos podrán ser más útiles en el ámbito de la asistencia pública de la salud a partir del momento en que su cultura profesional comience a fortalecer modelos de intervención de más amplio alcance en el que puedan ser reconocidos como trabajadores de la salud pública preocupados por la promoción del bienestar de la población.

\section{SYNOPSIS}

\section{Misdirections in the professional training used to prepare psychologists in Brazil to work in the public health-care system}

\begin{abstract}
In Brazil, the practice of psychologists in public health-care institutions is hampered by the professional training that they have received. That training is inadequate in meeting the requirements of primary health care, in satisfying the needs of the patients who use the public health-care system, and in overcoming the social inequalities that exist in Brazil. This piece is intended to identify the factors that make it harder to provide adequate psychological care in public health-care institutions, especially in the local Basic Health Units of Brazil's public health-care system, the Unified Health System. These hindrances to providing good care mainly originate in training that points psychologists in the direction of very restricted models of health care. These narrow models prevent the psychologists from responding appropriately to the needs of their patient clientele and of the public institutions. These limited models also make it hard for psychologists to adapt to the dynamic conditions that they will face in the Unified Health System.
\end{abstract}

\section{REFERENCIAS}

1. Boarini ML. A formação (necessária) do psicólogo para atuar na saúde pública. Psicologia em Estudo 1996;1(1):93-132.

2. Boarini ML. O psicólogo e a UBS: o desvio de rumo En: Zanella, A et al, orgs. Psicologia e Práticas Sociais. Porto Alegre: ABRAPSOSUL 1997; Pp.399-409.

3. Dimenstein M. O psicólogo no contexto do sistema único de saúde (SUS): Perfil profissional e perspectivas de atuação nas unidades básicas de saúde (UBS). [Tese de doutorado]. Rio de Janeiro: Instituto de Psiquiatria, Universidade Federal do Rio de Janeiro; 1998.
4. Dimenstein M. O psicólogo e o compromisso social no contexto da saúde coletiva. Psicologia em Estudo (Maringá) 2001;6(2):57-63.

5. Dimenstein M. O psicólogo nas unidades básicas de saúde: desafios para a formação e atuação profissionais. Estudos de Psicología (Natal) 1998;3(1):53-82.

6. Yamamoto OH. Trinta anos de SEPA, trinta anos de psicologia no Brasil. Estudos de Psicologia 1996;1(1):92-107.

7. Minayo MCS. O desafio do conhecimento: pesquisa qualitativa em saúde. São Paulo, Rio de Janeiro: Hucitec/ Abrasco; 1992.

8. Minayo MCS. Saúde e doença como expressão cultural. En: Amâncio Filho A, org. Saúde, trabalho e formação profissional. Rio de Janeiro: Fiocruz; 1997. Pp. 31-40.

9. Brasil, Ministério da Saúde, VIII Conferência Nacional de Saúde, Relatório Final, Brasília, 1986.

10. Vasconcelos EM. Educação popular nos serviços de saúde. São Paulo: Hucitec; 1991. 